\title{
Adaptação cultural e validação do instrumento Demandas de Atenção Dirigida*
}

\author{
CULTURALADAPTATION AND VALIDATION OF THE INSTRUMENT REQUIREMENT FOR \\ DIRECTEDATTENTION
}

ADAPTACIÓN CULTURAL Y VALIDEZ DEL INSTRUMENTO DEMANDAS DE ATENCIÓN DIRIGIDA

\section{Edinêis de Brito Guirardello ${ }^{1}$}

\section{RESUMO}

Este estudo tem como objetivos traduzir e validar o instrumento Requirement for Directed Attention (RDA), desenvolvido por Guirardello, para a cultura brasileira. É um instrumento composto de 39 itens que aborda situações no ambiente de trabalho de unidades de cuidados críticos e médicocirúrgica que demandam atenção dirigida do enfermeiro. $\mathrm{O}$ procedimento metodológico constituiu nas etapas de: a) tradução do instrumento para a língua portuguesa; b) back-translation; c) avaliação por um grupo de juízes e d) pré-teste da versão final do instrumento. $\mathrm{O}$ instrumento na versão em Português foi aplicado a um grupo de 44 enfermeiros. Os dados resultaram em alta consistência interna para o total dos itens $(\alpha=0,91)$ e para os domínios: Ambiente Físico $(\alpha=0,78)$, Psicológico $(\alpha=0,85)$ e Comportamental $(\alpha=0,80)$. Apenas o domínio Informação obteve um a de 0,12 . Esses resultados sugerem que a versão em Português do RDA pode ser usada para avaliar as demandas de atenção do enfermeiro.

\section{DESCRITORES}

Psicometria (normas).

Atenção.

Cuidados críticos.

Validade.

\begin{abstract}
The aim of this study is to translate and validate the instrument Requirement for Directed Attention (RDA), developed by Guirardello, into Brazilian culture. The instrument, comprised of 39 items, addresses situations in the work environment of critical care and medical-surgical units that demand the nurse's direct attention. The methodological approach consists of the following steps: a) translation of the instrument to the Portuguese language; b) backtranslation; c) committee review; and d) pre-testing of the instrument's final version. The Portuguese language version was applied to 44 nurses. The data resulted in high internal consistency for the total of the items $(\alpha=0.91)$, and for the domains: Physical Environment $(\alpha=0.78)$, Psychological Envi-ronment $(\alpha=0.85)$ and the Behavioral Environment $(\alpha=0.80)$. Only the domain Information had a low value $(\alpha=0.12)$. These results suggest that the Portuguese language version of the RDA can be used to evaluate the attention demands for the nurse.
\end{abstract}

\section{KEY WORDS}

Psychometrics (standards).

Attention.

Critical care.

Validity.

\section{RESUMEN}

Este estudio tuvo como objetivo traducir y validar la metodología Requirement for Directed Attention (RDA) desarrollado, para la cultura brasileña, por Guirardello. El instrumento está compuesto por 39 situaciones del ambiente de trabajo en unidades de cuidados críticos y médico-quirúrgicos que requieren la atención dirigida del enfermero. Para el procedimiento metodológico se siguieron las siguientes etapas: a) traducción del instrumento al idioma portugués; b) retrotraducción; c) evaluación de su equivalencia y d) prueba piloto. El instrumento, en el idioma portugués, fue aplicado a un grupo de 44 enfermeros. Los resultados muestran una alta consistencia interna para el total de los items $(\alpha=0,91)$ y para los dominios: Ambiente Físico $(\alpha=0,78)$, Psicológico $(\alpha=0,85)$ y Comportamental $(\alpha=0,80)$. El dominio información obtuvo apenas una a de 0,12 para el cual fue obtenido un $\alpha=0,12$. Estos resultados sugieren que la versión en portugués del RDA, puede ser usada para evaluación de las demandas para la atención del enfermero.

\section{DESCRIPTORES}

Psicometría (normas).

Atención.

Cuidados críticos.

Validez.
* Projeto financiado pela FAPESP n. 2000/11845-1

1 Professora Doutora do Departamento de Enfermagem da Faculdade de Ciências Médicas da Unicamp (FCM/UNICAMP). guirar@fcm.unicamp.br 


\section{INTRODUÇÃO}

A atenção é necessária para que o enfermeiro possa processar informações, planejar e prestar assistência, bem como estar atento às possíveis intercorrências, apresentadas por pacientes em risco de vida e com alto grau de complexidade de cuidados.

Na prática diária, o enfermeiro de unidades de cuidados críticos deve direcionar atenção para múltiplas e frequentemente competitivas demandas impostas pela grande variedade de estímulos no ambiente, enquanto presta assistência aos pacientes e a seus familiares, ao mesmo tempo em que se relaciona com colegas e outros profissionais. Esses estímulos podem ser identificados como sobrecarga de trabalho, conflito com outros colegas, dificuldades relacionadas com a assistência ao paciente criticamente doente, iluminação inadequada, barulho excessivo ou silêncio total ${ }^{(1-4)}$.

Essas situações, vivenciadas pelo enfermeiro, podem resultar em um esforço mental significativo para o desempenho de suas atividades. Além disso, demandas existentes em seu trabalho, como também recursos insuficientes para atender as necessidades e objetivos, possibilitam exceder a capacidade de direcionar atenção (CDA) do enfermeiro, podendo assim afetar a qualidade da assistência fornecida aos pacientes e seus familiares.

A CDA é um tipo de atenção seletiva, originalmente referida como atenção voluntária. Atenção é a concentração da mente em um objeto ou um pensamento, apesar de vários outros estímulos ocorrerem simultaneamente ${ }^{(5)}$. A capacidade de direcionar atenção é a habilidade do indivíduo em inibir estímulos e distrações competitivas, tanto do ambiente interno como do ambiente externo, enquanto processa e organiza uma informação importante e isto requer o uso de esforço mental. É enfatizado que a CDA é necessária para o funcionamento efetivo da vida diária, permitindo a uma pessoa perceber, pensar com clareza e manter atividade intencionada apesar de distrações que se originam do ambiente ${ }^{(6-7)}$.

O uso intenso e prolongado da CDA pode resultar em fadiga de atenção, que é caracterizada por uma diminuição na capacidade de manter o foco e concentrar-se. Ou seja, a pessoa apresenta dificuldades para direcionar atenção para novas informações, para estabelecer objetivo, para resolver problemas e realizar suas atividades diárias ${ }^{(8)}$.
$\mathrm{Na}$ tentativa de conduzir atividades práticas ou significativas frente à estímulos distrativos como barulho no ambiente externo ou preocupações no ambiente interno, os indivíduos devem dispender maior esforço inibitório para suprimir atividades cognitivas competitivas para manter claridade de foco e agir efetivamente.

O enfermeiro de unidades de cuidados críticos está em risco para desenvolver a fadiga de atenção, pois no dia a dia de seu trabalho necessita manter foco ou concentrar-se em atividades essenciais que envolvem o cuidado ao paciente, e muitas vezes essas demandas podem exceder a sua capacidade de direcionar atenção, podendo resultar em um esforço mental significativo para o desempenho de suas atividades.

$\mathrm{O}$ enfermeiro pode vivenciar muitas demandas para atenção devido a: atividades de trabalho que requerem constante vigilância dos pacientes e processamento de grande quantidade de informação na prestação do cuidado; ambiente de trabalho com excesso de barulho, ausência de iluminação natural e acúmulo de pessoas no local; fatores emocionais como observar o sofrimento do paciente e necessidade de dar suporte emocional aos familiares e pacientes; e fatores interpessoais como falta de oportunidade para expressar e compartilhar sentimentos com outras pessoas na unidade.

Destaca-se quanto a importância de utilizar instrumentos de medida para identificar os fatores no ambiente que podem aumentar as demandas para atenção, como também encontrar meios para conservar e recuperar a capacidade de direcionar atenção.

A autora ${ }^{(9)}$ desenvolveu um instrumento com o objetivo de identificar as situações usualmente vivenciandas pelo enfermeiro no seu ambiente de trabalho e que requerem o uso da atenção dirigida ou esforço metal. Os dados mostraram que ambos os grupos de enfermeiros (UTI e clínica médica e cirúrgica) vivenciam múltiplas situações no seu ambiente de trabalho que aumentam as necessidades para o uso da atenção dirigida, sendo essas relacionadas aos fatores psicológicos, comportamentais, ambiente físico e de informação. Considerando a inexistência de pesquisas no Brasil, enfocando tais questões, este estudo teve como objetivo traduzir e validar o instrumento Requirement for Directed Attention (RDA), para a língua portuguesa.
Rev Esc Enferm USP 2005; 39(1):77-84. 
TRAJETÓRIA METODOLÓGICA

\section{Adaptação cultural do instrumento RDA}

O processo de tradução e adaptação cultural do instrumento teve por base as recomendações de diversos pesquisadores ${ }^{(10-12)} \mathrm{e}$ envolvem as etapas de a) tradução do instrumento para o português; b) back-translation ou tradução da versão de volta para o inglês; c) avaliação por um grupo de juízes e d) pré-teste da versão final. Segundo esses pesquisadores, são passos essenciais que devem envolver o processo de tradução e adaptação de instrumentos.

\section{A escolha do instrumento}

$\mathrm{O}$ instrumento $R D A$ foi selecionado para este estudo, por se tratar de um instrumento desenvolvido pela autora, como pré-requisito para a sua dissertação de mestrado, intitulado Factors in the critical care and medical-surgical environments that increase requirements for directed attention ${ }^{(9)}$ e que necessita de avaliação de sua propriedade psicométrica. Esse instrumento foi desenvolvido com base no referencial teórico de Atenção e Demandas de Atenção ${ }^{(6,13-14)}$ para identificar os fatores no ambiente de trabalho que requerem atenção dirigida do enfermeiro.

O RDA contém 39 itens agrupados em quatro domínios: Ambiente Físico, Psicológico, Comportamental e de Informação. O domínio Ambiente Físico, com 12 itens, aborda fatores no ambiente externo, que causa na pessoa um aumento do esforço mental para conduzir uma determinada atividade. O Psicológico contém 12 itens, envolvendo emoções e preocupações que funcionam como fonte de distração. $\mathrm{O}$ domínio Comporta-mental aborda 12 itens de natureza física, social ou situacional que interfere com ou restringe as inclinações da pessoa. O domínio Informação, com três itens, envolve fatores que interferem com a percepção e interpretação de uma informação.

Cada item possui dois tipos de medidas: a primeira questiona sobre a freqüência com que se vivenciou determinada situação, representada numa escala do tipo Likert, de zero a quatro pontos, variando de nenhuma vez para muitas vezes ou o tempo todo, e a segunda, a intensidade, ou seja, o quanto de esforço mental foi necessário para lidar com aquela situação, representada por meio de uma escala analógica visual de zero a $100 \mathrm{~mm}$, com dois extremos (sem esforço/nada exigente para extremo esforço/ extremamente exigente).

\section{Tradução para língua portuguesa}

Essa etapa consistiu na tradução da versão original do instrumento para a língua portuguesa, por dois tradutores independentes, cuja língua materna é o Português, resultando assim em duas versões. A seguir, as duas versões foram avaliadas individualmente, e depois comparadas uma com a outra por dois outros pesquisadores, para obtenção de uma única versão em português. Isto permitiu a detecção de erros e interpretações divergentes de itens com significados ambíguos na versão original ${ }^{(10)}$.

\section{Tradução do instrumento de volta para o idioma de origem}

Também denominada de back-translation, consiste em traduzir o conteúdo do instrumento na segunda língua para o idioma de origem. Participaram dessa etapa dois tradutores independentes cuja língua materna é o inglês, para realizar a tradução do instrumento de volta para o inglês, resultando assim em duas versões. Em seguida as duas versões foram avaliadas individualmente e comparadas com o original em inglês e as discrepâncias foram analisadas. Esse procedimento permite avaliar se a versão em português corresponde adequadamente à versão em inglês.

\section{Avaliação por um grupo de juízes}

Após a obtenção da versão final traduzida para o português, o próximo passo metodológico consistiu na validação da mesma por um grupo de juízes, caracterizando-se assim a terceira etapa do processo de adaptação cultural do instrumento.

Os juízes foram solicitados a avaliar as equivalências semântica e idiomática e equivalências cultural e conceitual do instrumento, por meio de instruções específicas ${ }^{(15)}$, sendo essas realizadas individualmente.

Na avaliação da equivalência semântica e idiomática do instrumento, participaram três juízes, sendo dois enfermeiros e um professor de inglês. Todos os juízes tinham domínio da língua e dois deles haviam morado nos Estados Unidos. Para avaliação da equivalência cultural e conceitual do instrumento, participaram quatro juízes, sendo todos pesquisadores, com experiência em validação de instrumentos de medida.

\section{Pré-teste da versão final do instrumento}

O pré-teste constituiu a última etapa do processo de adaptação cultural. Após os ajustes necessários, a versão final do instrumento foi sub-
Adaptação cultural

e validação do

instrumento

Demandas de

Atenção Dirigida 
metida a um pré-teste para análise da compreensibilidade dos itens. Optou-se pela técnica de pro$\mathrm{va}^{(10)}$ que consiste na determinação da compreensibilidade do instrumento a ser aplicado a uma amostra da população-alvo. Esta técnica permite avaliar a validade aparente (validade de conteúdo) do instrumento adaptado.

Participaram do pré-teste oito enfermeiros que trabalham em unidades de cuidados críticos. As instruções sobre o preenchimento do instrumento assim como cada um dos itens, foram discutidos com os enfermeiros quanto à compreensão e clareza.

Após essa etapa, o instrumento Requirement for Directed Attetion passou a ser denominado de Demandas de Atenção Dirigida, apresentado no Quadro 1, apresentado na sequência.

\section{Quadro 1- Demandas de Atenção Dirigida}

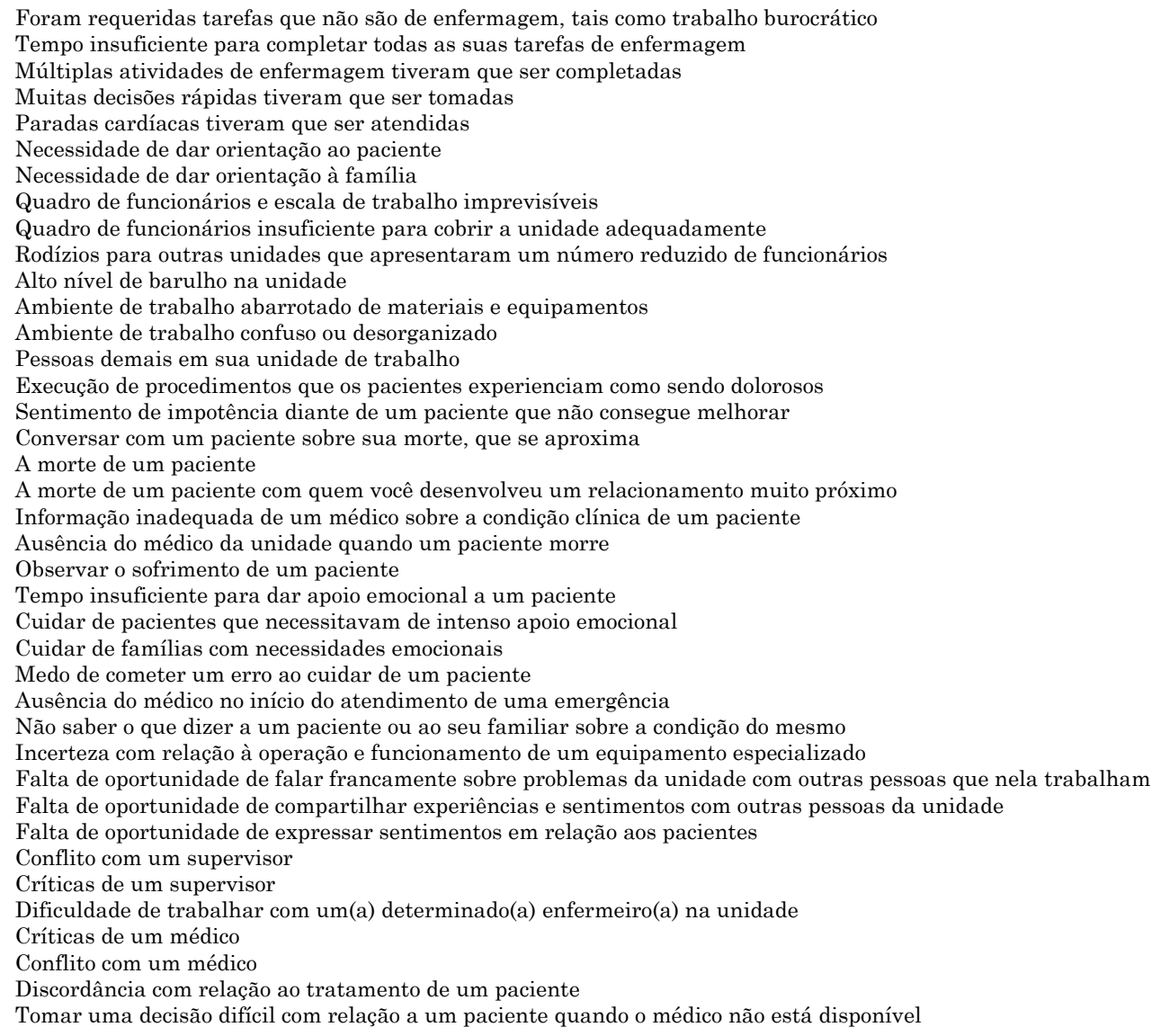

\section{Tratamento dos dados}

Para a análise estatística dos dados, utilizouse o programa computacional "the SAS System for Windows" (Statistical Analysis System). Para analisar a concordância das respostas dos juízes para todos os itens do instrumento, foi utilizado o teste Qui-Quadrado de Cochran para respostas dicotômicas ${ }^{(16)}$. Para quantificar o grau de concordância entre os três juízes foi calculado o coeficiente Kappa de concordância múltipla ${ }^{(17)}$.

Para cada item foi calculado o Índice de Validade de Conteúdo (IVC), que indica, item a item, qual a porcentagem de respostas com tradução satisfatória entre os juízes. Este índice auxilia na identificação dos possíveis itens que estejam necessitando de alterações na fase de validação e adaptação do instrumento.

A estatística descritiva foi utilizada para descrever o perfil da amostra segundo as diversas variáveis em estudo, com cálculo da média aritmética, desvio-padrão e mediana das variáveis contínuas.

Para analisar a consistência interna das respostas aos itens do instrumento, foi calculado o coeficiente de Cronbach $^{(18-19)}$. Valores acima de 0,80 indicam alta consistência interna, enquanto que valores acima de 0,60 indicam consistência intermediária. 


\section{Avaliação das propriedades psicométricas do instrumento}

Esta etapa compreendeu a avaliação da confiabilidade e validade do instrumento adaptado. Sugere-se que sejam feitas validações de conteúdo, de critério e de conceito e confiabilidade intra e inter observadores ${ }^{(8)}$.

A confiabilidade de um instrumento é o grau de coerência com o qual o instrumento mede um atributo. Quanto menor a variação produzida por um instrumento aplicado repetidamente, maior será sua confiabilidade.

A validade de um instrumento refere-se ao aspecto da medida ser congruente com a propriedade medida dos objetos e não a exatidão com que a mensuração que descreve esta propriedade é realizada ${ }^{(15)}$.

\section{Local do estudo}

O estudo das propriedades psicométricas do instrumento Demandas de Atenção Dirigida foi realizado mediante aplicação deste instrumento em duas unidades de cuidados críticos de um hospital-escola no interior do Estado de São Paulo. A primeira unidade, denominada de Unidade de Terapia Intensiva (UTI), com capacidade de atendimento para 18 pacientes e a segunda, denominada de Unidades de Emergência Clínica e Cirurgia do Trauma (EE/CT), tem capacidade de atendimento para 30 pacientes. A unidade de Emergência Clínica tem 14 leitos, sendo seis intensivos e oito semi-intensivos e a unidade de Cirurgia do Trauma tem 16 leitos, sendo 12 semi-intensivos e quatro intensivos

\section{População}

A população do estudo constituiu-se de enfermeiros que exercem atividades assistenciais, provenientes de duas unidades de cuidado críticos (UTI e EC/CT) de um hospital escola, do interior do Estado de São Paulo.

A UTI contava, no momento em que o estudo foi feito, com 32 enfermeiros, porém três enfermeiros não foram abordados nesta etapa por terem participado da etapa do pré-teste. Do total de 29 enfermeiros, um estava de férias; outro retornou o instrumento em branco e um outro não devolveu o instrumento.

A unidade de EC/CT contava no seu quadro de pessoal com 21 enfermeiros. Desses, obtivemos a participação de 19 enfermeiros, mas foram considerados na amostra apenas 18, pois um instrumento foi cancelado por erros no seu preenchimento e um enfermeiro não retornou $\mathrm{o}$ instrumento.

\section{Instrumento de coleta de dados}

Os instrumentos utilizados para a coleta de dados foram o instrumento de Demandas de Atenção Dirigida e uma ficha com dados de identificação do enfermeiro. A ficha de identificação contém dados como: idade, sexo, tempo de formação profissional, tempo de experiência na unidade, carga horária, local e turno de trabalho.

\section{Procedimento para coleta de dados}

Primeiramente, este projeto obteve autorização do Comitê de Ética em Pesquisa da Faculdade de Ciências Médicas da Universidade Estadual de Campinas (Unicamp). A seguir, foi realizado contato com a diretora de enfermagem de ambas as unidades UTI e EC/CT a qual foi solicitada autorização para desenvolvimento da pesquisa e acesso às escalas de trabalho. Os enfermeiros foram convidados a participar do estudo e, após sua anuência, foram solicitados a assinar o Termo de Consentimento Livre e Esclarecido.

Em seguida, foram orientados quanto ao preenchimento do instrumento. $\mathrm{O}$ instrumento, a ficha contendo informações gerais como idade, sexo, tempo de formação, carga horária, local e turno de trabalho, bem como o termo de consentimento, foram entregues para o enfermeiro dentro de um envelope. O retorno do mesmo foi combinado previamente com cada um dos participantes. A coleta de dados ocorreu no próprio local de trabalho do participante, sendo esta realizada pela própria pesquisadora.

\section{RESULTADOS E DISCUSSÃO}

\section{Avaliação das equivalências pelo grupo de juízes}

$\mathrm{Na}$ avaliação da equivalência semântica e idiomática do instrumento, os dados mostraram que não houve discordância significativa entre os três juízes (Cochran's Q =4,67, p=0,097). Um dos juízes aprovou 34 itens satisfatórios e cinco insatisfatórios. Um outro juiz aprovou 34 itens satisfatórios e seis insatisfatórios. O terceiro juiz aprovou 38 itens satisfatórios e um insatisfatório. Quando calculado o coeficiente de concordância múltipla, houve concordância significativa entre os juízes, embora o valor de Kappa tenha sido baixo $(\mathrm{K}=0,16)$.

Para o cálculo do índice de validade de conteúdo (IVC), os itens com baixo IVC foram: 6, 7 e 34
Adaptação cultural

e validação do

instrumento

Demandas de

Atenção Dirigida 
$(\mathrm{IVC}=33,3)$ e itens $3,10,12,27,28 \mathrm{e} 36(\mathrm{IVC}=66,7)$. Por exemplo: nos itens 6 e 7 , foi sugerido a substituição da palavra requerida para necessária. No item "críticas por um(a) supervisor(a)" foi sugerida substituir para "críticas feitas por um supervisor".

$\mathrm{Na}$ avaliação da equivalência cultural e conceitual, os resultados dos testes de concordância entre os juízes mostraram que não houve discordância significativa entre os mesmos (Cochran's $\mathrm{Q}=6,33, \mathrm{p}=0,096$ ). Dois juízes aprovaram 38 itens satisfatórios e um insatisfatório. Outro juiz aprovou 36 itens satisfatórios e três insatisfatórios. Um outro juiz aprovou todos os itens. Quando calculado o coeficiente de concordância múltipla, houve concordância significativa entre os juízes $(\mathrm{K}=0,38, \mathrm{p}=0,001)$.
No cálculo do índice de validade de conteúdo (IVC) para cada item, a análise mostrou que apenas a situação "ambiente de trabalho cheio", apresentou baixo IVC $(25,0)$.

\section{Caracterização dos enfermeiros}

Participaram do estudo 44 enfermeiros, sendo 26 enfermeiros da UTI e 18 enfermeiros das unidades de EC e CT de um hospital-escola no interior do Estado de São Paulo. Para descrever o perfil da amostra segundo as diversas variáveis em estudo, foram feitas tabelas de frequência das variáveis categóricas e estatísticas descritivas das variáveis contínuas. Esses dados estão apresentados na Tabela 1.

Tabela 1- Características sociodemográficas dos enfermeiros. (Campinas, 2003)

\begin{tabular}{|c|c|c|}
\hline \multirow{2}{*}{$\begin{array}{c}\text { Características } \\
\text { Sexo }\end{array}$} & \multicolumn{2}{|c|}{ Distribuição da amostra } \\
\hline & $\mathbf{n}$ & $\%$ \\
\hline $\begin{array}{c}\text { Feminino } \\
\text { Masculino }\end{array}$ & $\begin{array}{l}34 \\
10\end{array}$ & $\begin{array}{l}(77,3 \%) \\
(22,7 \%)\end{array}$ \\
\hline \multicolumn{3}{|l|}{ Idade (anos) } \\
\hline $\begin{array}{c}20-29 \\
30-39 \\
40-49 \\
>50\end{array}$ & $\begin{array}{c}13 \\
18 \\
12 \\
1\end{array}$ & $\begin{array}{c}(29,5 \%) \\
(40,9 \%) \\
(27,3 \%) \\
(2,3 \%)\end{array}$ \\
\hline \multicolumn{3}{|l|}{ Estado Civil } \\
\hline $\begin{array}{l}\text { Solteiro } \\
\text { Casado } \\
\text { Divorciado } \\
\text { Outros }\end{array}$ & $\begin{array}{l}25 \\
15 \\
2 \\
2\end{array}$ & $\begin{array}{c}(56,8 \%) \\
(34,1 \%) \\
(4,5 \%) \\
(4,5 \%)\end{array}$ \\
\hline \multicolumn{3}{|l|}{ Turno de trabalho } \\
\hline $\begin{array}{l}\text { Manhã } \\
\text { Tarde } \\
\text { Noite } \\
\text { Outros }\end{array}$ & $\begin{array}{c}7 \\
11 \\
17 \\
9\end{array}$ & $\begin{array}{l}(15,9 \%) \\
(25,0 \%) \\
(38,6 \%) \\
(20,5 \%)\end{array}$ \\
\hline \multicolumn{3}{|c|}{ Experiência profissional (anos) } \\
\hline $\begin{array}{c}0-10 \\
11-20 \\
21-30\end{array}$ & $\begin{array}{c}28 \\
12 \\
4\end{array}$ & $\begin{array}{c}(63,6 \%) \\
(27,3 \%) \\
(9,1 \%)\end{array}$ \\
\hline \multicolumn{3}{|c|}{ Horas de trabalho/ últ. semana } \\
\hline $\begin{array}{c}<06 \text { a } 12 \mathrm{~h} \\
24 \text { a } 36 \mathrm{~h} \\
40 \text { a } 48 \mathrm{~h}\end{array}$ & $\begin{array}{c}1 \\
26 \\
17\end{array}$ & $\begin{array}{l}(2,3 \%) \\
(59,1 \%) \\
(38,6 \%)\end{array}$ \\
\hline
\end{tabular}

De acordo com a Tabela 1, verifica-se que a maioria dos participantes (77,3\%) é do sexo feminino. A média de idade dos participantes foi de 34,7 anos, sendo a maioria solteira e com um tempo de experiência profissional de dez anos.

Com a análise dos dados, foi possível verificar que a amostra foi homogênea com relação ao sexo (Fisher, $\mathrm{p}=0,72$ ) e idade (Mann-Whitney, $p=0,78)$. Não houve diferenças significativas no que se referiu ao tempo de experiência (Fisher, $\mathrm{p}=0,36$ ); turno (Fisher, $\mathrm{p}=0,21$ ) e horas de trabalho $\left(x^{2}=0,51, \mathrm{p}=0,77\right)$.

\section{Análise da consistência interna do instrumento}

A confiabilidade da versão final do instrumento foi avaliada pela consistência interna das respostas aos itens do instrumento, com o cálculo do coeficiente a de Cronbach. Conforme Tabela 2, apresentam-se os coeficientes a de Cronbach para o escore conjunto, para todos os itens do instrumento e para cada um dos quatro domínios (Ambiente físico, Comportamental, Psicológico e Informação). 
Tabela 2 - Consistência interna dos itens e domínios do instrumento. (Campinas, 2003)

\begin{tabular}{lccccc}
\hline \multicolumn{1}{c}{ Domínios } & $\begin{array}{c}\mathrm{N}^{\mathrm{o}} \mathrm{de} \\
\text { Itens }\end{array}$ & de Cronbach & $\begin{array}{c}\text { Itens com } \\
\text { menor } \\
\text { consistência }\end{array}$ & $\begin{array}{c}\text { Correlação } \\
\text { do item } \\
\text { com o total }\end{array}$ & $\begin{array}{c}\text { de Cronbach, } \\
\text { após retirada } \\
\text { dos itens }\end{array}$ \\
\hline $\begin{array}{l}\text { Ambiente físico } \\
\text { Comportamental }\end{array}$ & 12 & $\mathbf{0 , 7 8}$ & 1 & 0,07 & $\mathbf{0 , 8 0}$ \\
Psicológico & 12 & $\mathbf{0 , 8 0}$ & 31 & 0,20 & $\mathbf{0 , 8 1}$ \\
Informação & 12 & $\mathbf{0 , 8 5}$ & 26 & 0,17 & $\mathbf{0 , 8 7}$ \\
\hline Total & 3 & $\mathbf{0 , 1 2}$ & 29 & $-0,02$ & $\mathbf{0 , 2 8}$ \\
\hline
\end{tabular}

Verifica-se alta consistência interna para o total dos itens $(\alpha=0,91)$ e para todos os domínios, exceto para o domínio "Informação" $(\alpha=0,12)$, provavelmente pelo número de itens do mesmo e pela pouca correlação entre os itens dentro desse domínio.

Os itens com menor consistência interna foram: "incerteza com relação à operação e funcionamento de um equipamento especializado" $(\alpha=-0,27)$, ou seja, inversamente proporcional. Para o item "foram requeridas tarefas que não são de enfermagem, tais como trabalho burocrático" $(\alpha=0,32)$.

\section{CONCLUSÕES}

A versão traduzida do RDA para o português atendeu aos critérios de equivalência semântica, idiomática, cultural e conceitual. $\mathrm{Na}$ análise das equivalências semântica e idiomática do instrumento, os dados mostraram não haver discordância significativa entre os juízes (Cochran's $\mathrm{Q}=4,67$, $\mathrm{p}=0,097$ ), e o cálculo do coeficiente de concordância múltipla resultou em concordância significativa entre os juízes, embora o valor de Kappa seja baixo $(K=0,16)$. Na avaliação da equivalência cultural e conceitual, os resultados mostraram não haver discordância significativa entre os juízes com relação aos itens do instrumento (Cochran's $\mathrm{Q}=6,33, \mathrm{p}=0,096$ ) e o coeficiente de concordância múltipla demonstrou existência de concordância significativa entre os juízes $(\mathrm{K}=0,38, \mathrm{p}=0,0001)$.
O instrumento na versão em português apresentou alta consistência interna para o total dos itens $(\alpha=0,91)$ e para os domínios Ambiente Físico ( $\alpha=0,78)$, Psicológico $(\alpha=0,85)$ e Comportamental $(\alpha=0,80)$, exceto para o domínio Informação, provavelmente pelo número de itens do mesmo e pela pouca correlação entre os itens desse domínio. Embora o domínio Informação tenha resultado em baixa consistência interna, optou-se por manter os itens nesse domínio, para posterior aplicação do instrumento em uma amostra mais representativa dessa população.

Concluiu-se que o instrumento Demandas de Atenção Dirigida pode ser aplicado em nosso meio, pois ele possibilita identificar os fatores no ambiente de trabalho que requerem o uso da atenção dirigida pelo enfermeiro. Recomenda-se, entretanto, a sua aplicação em outras populações com características semelhantes para testar os itens que não foram consistentes na amostra estudada.

A utilização desse instrumento possui importantes implicações para enfermeiros, gerentes de enfermagem e administradores e pode ser usado pelos administradores de enfermagem para identificar os fatores de demanda para atenção dirigida no ambiente de trabalho do enfermeiro. Desta forma, os administradores, gerentes e enfermeiros podem estabelecer estratégias que minimizem essas demandas.

\section{REFERÊNCIAS}

(1) Huckabay LM, Jagla B. Nurses' stress factors in the intensive care unit. J Nurs Adm 1979; 9(2):21-6.

(2) Gray-Topt P, Anderson JG. Stress among hospital nursing staff: its causes and effects. Soc Sci Med 1981;15(5):639-47.

(3) Norbeck JS. Perceived job stress, job satisfaction, and psychological symptoms in critical care nursing. Res Nurs Health 1985; 8(3):253-9.

(4) Dewe PJ Identifying the causes of nurses' stress: a survey of New Zealand nurses. Work \& Stress 1987; 1(1):15-21.
(5) James W. The principles of psychology. Cambridge: Dover Publications; 1950.

(6) Cimprich B. Attentional fatigue following breast cancer surgery. Res Nurs Health 1992; 15(3):199-207.

(7) Cimprich B. Age and extend surgery affect attention in women treated for breast cancer. Res Nurs Health 1998; 21:229-38.

(8) Cimprich B. Attentional fatigue and restoration in individuals with cancer. [thesis] Michigan (MI): University of Michigan; 1990.
Rev Esc Enferm USP 2005; 39(1):77-84. 
(9) Guirardello EB. Factors in the critical care and medical surgical environment that increase the requirements for directed attention. [dissertation] Madison (WI): School of Nursing; 1993.

(10) Guillemin F, Bombardier C, Beaton D. Crosscultural adaptation of health-related quality of life measures: literature review and proposed guidelines. J Clin Epidemiol 1993; 46(12):1417-32.

(11) Hutchinson A, Bentzen N, Konig-Zanhn C. Crosscultural health-outcome assessment: a user's guide. The Netherlands: ERGHO; 1996.

(12) Guillemin F. Cross-cultural adaptation and validation of health status measures. Scand J Rheumatol 1995; 24(2):61-3.

(13) Kaplan S, Kaplan R. Cognition and environment: functioning in an uncertain world. New York: Praeger; 1983.
(14) Cimprich B. A theoretical perspective on attention and patient education. Adv Nurs Sci, 1992; 14(3):39-51.

(15) Kimura M. Tradução para o português e validação do "Quality of Life Index", de Ferrans e Powers. [livre-docência] São Paulo (SP): Escola de Enfermagem da USP; 1999.

(16) Conover WJ. Practical nonparametric statistics. New York: John Wiley; 1971.

(17) Fleiss JL. Statistical methods for rates and proportions. $2^{\text {nd }}$ ed. New York: John Wiley; 1981.

(18) Nunnally JC. Psychometric theory. $2^{\text {nd }}$ ed. New York: McGraw-Hill; 1978.

(19) Pereira JCR. Análise de dados qualitativos - estratégias metodológicas para as ciências da saúde, humanas e sociais. São Paulo: EDUSP; 1999.
Correspondência: Edinêis de Brito Guirardello Rua Catharina Signori Vicenti, 74

- Jardim Barão Geraldo - Campinas - São Paulo Cep: 13083-790

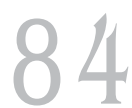

Rev Esc Enferm USP 2005; 39(1):77-84. 